\title{
The evaluation of iron content in Alzheimer's disease by magnetic resonance imaging: Phase and R2* methods
}

\author{
Bijing Zhou, Siyao Li, Huijin He*, Xiaoyuan Feng \\ Department of Radiology, Huashan Hospital Fudan University, Shanghai, China; ${ }^{*}$ Corresponding Author: huashansci2013@126.com
}

Received 21 February 2013; revised 28 March 2013; accepted 6 April 2013

Copyright (C) 2013 Bijing Zhou et al. This is an open access article distributed under the Creative Commons Attribution License, which permits unrestricted use, distribution, and reproduction in any medium, provided the original work is properly cited.

\begin{abstract}
Background: Magnetic resonance imaging (MRI) is the best imaging examination to evaluate $a b-$ normal iron deposition in the brain. Although phase of susceptibility weighted imaging (SWI) and $\mathrm{R2}^{*}$ values have been used to probe iron deposition in Alzheimer's disease (AD) brain, no study has exploited both techniques for quantification of iron deposition in AD. Purpose: Use phase and $R 2^{*}$ to evaluate iron changes in $A D$ brain. Investigate the correlation of two methods with the severity of cognitive impairment in $A D$ patients. Materials and methods: Twenty-three patients with $A D$ and eighteen normal controls underwent SWI and multi-echo gradient recalled-echo (GRE) imaging on a 3T MR scanner. The phase values from SWI and $\mathrm{R2}^{*}$ values calculated from multi-echo GRE imaging of bilateral hippocampus, globus pallidus, putamen, caudate nucleus, thalamus, substantia nigra, red nucleus and dentate nucleus were evaluated. Results: In AD group, the phase values of bilateral hippocampus, globus pallidus, caudate nucleus, substantia nigra and left putamen were significantly lower than the control group. The $\mathrm{R2}^{*}$ values of bilateral hippocampus, caudate nucleus, putamen and right globus pallidus were significantly higher than the control group. The phase and $\mathbf{R 2}^{*}$ values of the left putamen had the most significant correlation with mini-mental state examination (MMSE) scores in AD patients. Conclusion: The SWI phase value and $\mathrm{R2}^{*}$ value can be used as effective methods to study the abnormality of iron deposition in $A D$ brain, wherein phase had advantages in small brain structure. Phase value showed a higher correlation coefficient with MMSE scores, moreover the
\end{abstract}

iron deposition of left putamen has a close relationship with the progression of AD.

Keywords: MMSE; Iron Deposition; SWI; Phase; Cognitive Impairment

\section{BACKGROUND}

Alzheimer's disease (AD) is an irreversible, progressive neurodegenerative disease, which is characterized pathologically by the generation of $\beta$-amyloid (A $\beta$ ) plaques and neurofibrillary tangles in the hippocampi and cerebral cortex. Iron deposition occurs in the brain in a variety of neurodegenerative disorders, such as $\mathrm{AD}$ and Parkinson's disease [1]. Oxidative damage to the brain of $\mathrm{AD}$ patients is one of the earliest pathological events [2, 3]. Increased redox active metal ions such as $\mathrm{Fe}^{3+}$ and $\mathrm{Cu}^{2+}$ can produce free radicals and predispose neurons to oxidative damage, also can induce increased generation of $\mathrm{A} \beta$ in $\mathrm{AD}$ brain [4], and when $\mathrm{A} \beta$ binds with $\mathrm{Fe}^{3+}$, it becomes aggregated and neurotoxic [5].

Magnetic resonance imaging (MRI) is the best imaging examination to evaluate abnormal iron deposition in the brain. It is non-invasive and non-radioactive and has advantages of the sensitivity of its signal to iron concentration. Several in vivo methods have been proposed for MRI detection and quantification of tissue iron, includeing R2 (1/T2), R2' (1/T2'), R2 ${ }^{*}\left(1 / \mathrm{T}^{*}\right)$, magnetic field correlation (MFC), field-dependent relaxation rate increase (FDRI) as well as susceptibility weighted imaging (SWI) [6-12]. Among them, R2 is highly dependent on complex relaxation mechanisms such as variation in types of tissues, experimental parameters, and magnetic field strength [13]. The FDRI requires more imaging time than SWI, two field strengths, and across-study image registration for iron concentration calculation. Additional measurement time and the difficulty in imaging registration are the disadvantages of the FDRI in clinical 
applications [14]. The SWI, based on a $\mathrm{T}^{*}$-weighted GRE sequence, utilizes phase information caused by different susceptibilities from tissues to enhance its contrast $[15,16]$ and has been proved to be a useful tool to quantify brain iron [17,18]. Although phase of SWI and $\mathrm{R} 2^{*}$ values have been used to probe brain iron in animal models and AD patients [19-21], no study has exploited both the techniques for quantification of iron deposition in neurodegenerative diseases.

In this prospective study, we used both phase of SWI and $\mathrm{R} 2{ }^{*}$ methods to assess iron concentration in $\mathrm{AD}$ brain. In addition, the correlation between iron content from each method and the severity of AD was compared.

\section{MATERIALS AND METHODS}

In this study, 23 patients (14 males, 9 females, mean age 70.5 years, range: 54 - 80 years) with $\mathrm{AD}$ were recruited from neurology department from August 2009 to January 2010. All AD patients were diagnosed by two neurologists according to the criteria of the National Institute of Neurological and Communication Disorders and Stroke/Alzheimer Disease and Related Disorders Association for probable AD [22]. The mini-mental state examination (MMSE) scores of the patients were 8 to 23 $(18.65 \pm 3.74)$. Participants with one of the following conditions were excluded: a) mental illness; b) any other diseases that could cause brain disorders and c) hyperintensities (diameter $\geq 2 \mathrm{~cm}$ ) on T2-weighted images. In addition, 18 age-and sex-matched normal subjects (10 male and 8 female) with mean age of 67.89 years (range: 59 - 76 years) and with MMSE scores ranging $28-30$ were recruited from the local community. All the normal control population had normal neurological findings and had no history of neurological disorders or head injuries or significant cerebral lesions except for lacunar infarcttion (diameter $<2 \mathrm{~cm}$ ) on conventional MR scan. The study was approved by the institutional review board of our hospital and all subjects were given written informed consent.

The MRI was performed on a 3.0 Tesla MR scanner (MAGNETOM Verio, Siemens Healthcare, Erlangen, Germany) equipped with a standard twelve-channel head coil. Conventional axial T1-weighted Turbo Spin Echo (TSE) images and Fluid Attenuate Inversion Recovery (FLAIR) sequence were acquired to screen cerebral lesions. Oblique T2-weighted TSE images perpendicular to the long axis of bilateral hippocampus were obtained to identify the hippocampi. The SWI images and $\mathrm{T}^{*}$ weighted multi-echo gradient recalled-echo (GRE) images were acquired by referencing this oblique T2weighted TSE plane to cover the exact volume from bilateral temporal poles to dentate nuclei (DN). For SWI, the following parameters was used: TR/TE $=28 / 20 \mathrm{~ms}$, flip angle $15^{\circ}$, the number of slices $=56$, slice thickness
$=2 \mathrm{~mm}, \mathrm{NEX}=1$, field of view $=230 \times 230 \mathrm{~mm}^{2}$, matrix size $=448 \times 448$, bandwidth $=120 \mathrm{~Hz} /$ pixel; for multi-echo GRE imaging: TR $=710 \mathrm{~ms}, 5$ echoes from $4.36 \mathrm{~ms}$ to $34.52 \mathrm{~ms}$ with an equal interval of $7.54 \mathrm{~ms}$ were used, flip angle $=60^{\circ}$, the number of slices $=56$, slice thickness $=2 \mathrm{~mm}, \mathrm{NEX}=1$, field of view $=230$ $\mathrm{mm} \times 230 \mathrm{~mm}$, matrix size $=384 \times 384$.

All data analysis were completed on phase images of SWI and slice-matched $\mathrm{T} 2^{*}$ maps, which were inline computed from $\mathrm{T} 22^{*}$-weighted multi-echo GRE images. For each region of interest (ROI) of each patient, like bilateral hippocampus (HP), globus pallidus (GP), putamen (PUT), caudate nucleus (CAU), thalamus (TH), substantia nigra (SN), red nucleus (RN) and $\mathrm{DN}$, two or three continuous slices from phase images of SWI that mainly covered the ROI were selected in consensus by two radiologists with sixteen and three years experience in Neuroradiology respectively, and then corresponding slices from $\mathrm{T}^{*}{ }^{*}$ map were chosen. In this process, CSF, bone and blood vessels were avoided to minimize the artifacts. Areas of ROI on each selected slice were manually identified and average values of right and left side ROIs were recorded respectively by the two radiologists on a commercial available workstation (Syngo, Siemens Healthcare) as demonstrated in (Figures 1-2). Both of them were blinded to the results of clinical diagnosis.

Since on Siemens scanner, the radius of phase is linearly rescaled from $(\pi,-\pi)$ to be $(-4096,4095)$ for contrast purpose, we returned the measured values on phase images to be in radius through the following formula:

$$
\phi=-\frac{M V}{4096} \pi
$$

where $\phi$ is the phase value in radius and $M V$ is the directly measured value on phase images.

For $\mathrm{R} 2{ }^{*}$ value, they were just transformed from $\mathrm{T} 2{ }^{*}$ by following the inversion relationship $\mathrm{R} 2^{*}=1 / \mathrm{T} 2^{*}$. Area weighted sum algorithm were applied on continuous selected slices, covering the same ROI, to calculate phase and $\mathrm{R} 2^{*}$ values as representative values on patient by patient basis.

Statistical analysis was performed by using software (SPSS for Windows, version 13.0; SPSS Inc., Chicago, IL). The ages and MMSE scores of two groups were analyzed using two tailed tests and the gender compareson between two groups was performed with Chi2-test. Phase values and $\mathrm{R} 2{ }^{*}$ values of each ROI in two groups were expressed as the mean $\pm \mathrm{SD}$. The comparison of phase values and $\mathrm{R} 2^{*}$ values of each ROI between two groups was performed with a nonparametric MannWhitney U test. A partial Spearman rank correlation coefficient, controlled by the age-related bias was used to assess the correlation between phase values and MMSE 


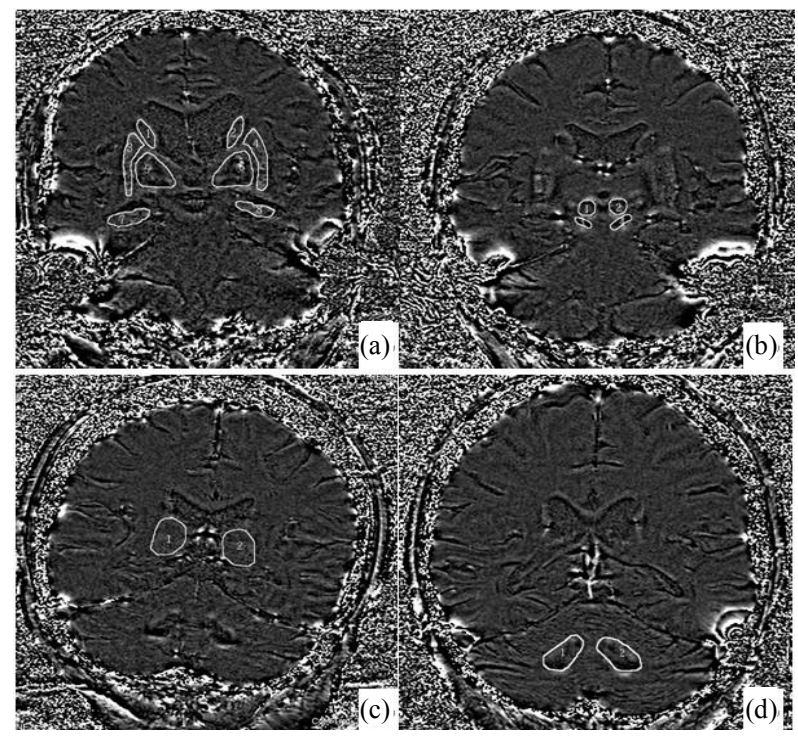

Figure 1. Phase images illustrate the selection of each region of interest (ROI). Note: (a) right caudate nucleus (1), left caudate nucleus (2), right putamen (3), left putamen (4), right globus pallidus (5), left globus pallidus (6), right hippocampus (7), left hippocampus (8). (b) right red nucleus (1), left red nucleus (2), right substantia nigra (3), left substantia nigra (4). (c) right thalamus (1), left thalamus (2). (d) Right dentate nuclei (1), left dentate nuclei (2).

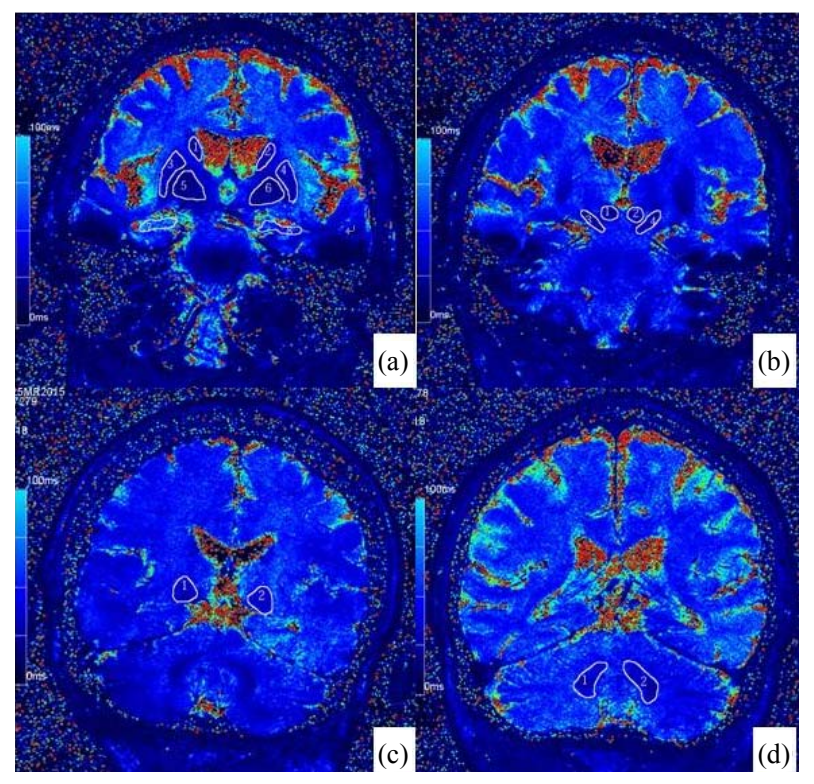

Figure 2. Caption: $\mathrm{R} 2^{*}$ images illustrate the selection of each ROI. Note: (a) right caudate nucleus (1), left caudate nucleus (2), right putamen (3), left putamen (4), right globus pallidus (5), left globus pallidus (6), right hippocampus (7), left hippocampus (8). (b) right red nucleus (1), left red nucleus (2), right substantia nigra (3), left substantia nigra (4). (c) Right thalamus (1), left thalamus (2). (d) Right dentate nuclei (1), left dentate nuclei (2).

scores as well as $\mathrm{R} 2^{*}$ values and MMSE scores in $\mathrm{AD}$ group. The results were considered statistically signifi- cant when $P<0.05$.

\section{RESULTS}

The demographic data for the $\mathrm{AD}$ and the control group were summarized in Table 1. The mean ages and gender of the two groups were statistically equal and the MMSE scores of the AD group were significantly lower than those of the control group $(P<0.05)$.

\subsection{The Phase Values of Right ROls}

Phase values of HP $(p=0.025)$, GP $(p=0.015)$, CAU $(\mathrm{p}=0.002)$, and $\mathrm{SN}(\mathrm{p}=0.005)$ and in $\mathrm{AD}$ group were significantly lower than the control group. However, the phase values of PUT $(-0.1160 \pm 0.0329$ vs. $-0.0983 \pm$ $0.0274 ; \mathrm{p}=0.098)$ and $\mathrm{DN}(-0.1127 \pm 0.0323$ vs. $-0.1166 \pm 0.0331 ; \mathrm{p}=0.854)$ were higher than the control group and not significant (Table 2, Figure 3(a)).

\subsection{The Phase Values of Left ROls}

Phase values of HP $(p=0.011)$, GP $(p=0.006)$, CAU $(p=0.001), S N(p=0.002)$ and PUT $(p=0.001)$ in AD group were significantly lower than the control group. However, the phase values of DN $(-0.1082 \pm 0.0288$ vs. $-0.1197 \pm 0.0429 ; \mathrm{p}=0.581)$ were higher than the control group and not significant (Table 2, Figure 3(b)).

\subsection{The $R 2^{*}$ Values of Right ROls}

Phase values of HP $(p=0.041)$, GP $(p=0.025)$, CAU $(p=0.039)$, and PUT $(p=0.027)$ in AD group were significantly higher than the control group (Table 3, Figure 3(c)).

\subsection{The R2* Values of Left ROls}

Phase values of HP $(p=0.001)$, CAU $(p=0.001)$, PUT ( $p=0.032)$, and DN (0.112) in AD group were significantly higher than the control group (Table 3, Figure 3(d)).

The phase values of bilateral GP, PUT, left HP, right $\mathrm{CAU}$ and right $\mathrm{DN}$ in $\mathrm{AD}$ group were correlated with MMSE scores and the correlation coefficients ranged

Table 1. Summary of demographics.

\begin{tabular}{ccccc}
\hline & AD & NC & Statistic value P value \\
\hline Number & 23 & 18 & & \\
$\begin{array}{c}\text { gender (male/female) } \\
\begin{array}{c}\text { age (y/o) } \\
(\text { mean } \pm \text { SD) }\end{array}\end{array}$ & $14 / 9$ & $10 / 8$ & $\chi^{2}=1.096$ & $\mathrm{P}>0.05$ \\
MMSE (mean \pm SD) & $18.65 \pm 3.7429 .11 \pm 1.21$ & $\mathrm{t}=10.10$ & $\mathrm{P}<0.05$ \\
\hline
\end{tabular}

$\mathrm{AD}=$ Alzheimer's disease $\mathrm{NC}=$ normal control $;$ MMSE $=$ Mini-Mental State Examination. 
Table 2. Phase value ( $\overline{\mathrm{X}} \pm \mathrm{s}$ ) of each ROI in $\mathrm{AD}$ and control group and Mann-Whitney U test.

\begin{tabular}{|c|c|c|c|c|c|}
\hline & ROI & $\mathrm{AD}$ & Control & $Z$ value & $P$ value \\
\hline \multirow[t]{2}{*}{ HP } & $\mathrm{R}$ & $-0.0951 \pm 0.0224$ & $-0.0636 \pm 0.0190$ & -2.243 & 0.025 \\
\hline & $\mathrm{L}$ & $-0.0913 \pm 0.0231$ & $-0.0588 \pm 0.0151$ & -2.473 & 0.011 \\
\hline \multirow[t]{2}{*}{ GP } & $\mathrm{R}$ & $-0.1501 \pm 0.0802$ & $-0.1012 \pm 0.0362$ & -2.443 & 0.015 \\
\hline & $\mathrm{L}$ & $-0.1537 \pm 0.0769$ & $-0.0999 \pm 0.0366$ & -2.732 & 0.006 \\
\hline \multirow[t]{2}{*}{ PUT } & $\mathrm{R}$ & $-0.1160 \pm 0.0329$ & $-0.0983 \pm 0.0274$ & -1.655 & 0.098 \\
\hline & $\mathrm{L}$ & $-0.1210 \pm 0.0377$ & $-0.0863 \pm 0.0198$ & -3.310 & 0.001 \\
\hline \multirow[t]{2}{*}{ CAU } & $\mathrm{R}$ & $-0.0938 \pm 0.0496$ & $-0.0564 \pm 0.0148$ & -3.100 & 0.002 \\
\hline & $\mathrm{L}$ & $-0.0878 \pm 0.0361$ & $-0.0586 \pm 0.0104$ & -3.389 & 0.001 \\
\hline \multirow[t]{2}{*}{$\mathrm{DN}$} & $\mathrm{R}$ & $-0.1127 \pm 0.0323$ & $-0.1166 \pm 0.0331$ & -0.184 & 0.854 \\
\hline & $\mathrm{L}$ & $-0.1082 \pm 0.0288$ & $-0.1197 \pm 0.0429$ & -0.552 & 0.581 \\
\hline \multirow[t]{2}{*}{$\mathrm{SN}$} & $\mathrm{R}$ & $-0.2090 \pm 0.0687$ & $-0.1418 \pm 0.0498$ & -2.837 & 0.005 \\
\hline & $\mathrm{L}$ & $-0.2151 \pm 0.0809$ & $-0.1396 \pm 0.0461$ & -3.126 & 0.002 \\
\hline \multirow[t]{2}{*}{$\mathrm{RN}$} & $\mathrm{R}$ & $-0.1436 \pm 0.0400$ & $-0.1249 \pm 0.0296$ & -1.786 & 0.074 \\
\hline & $\mathrm{L}$ & $-0.1366 \pm 0.0414$ & $-0.1167 \pm 0.0280$ & -1.629 & 0.013 \\
\hline \multirow[t]{2}{*}{$\mathrm{TH}$} & $\mathrm{R}$ & $-0.0510 \pm 0.0224$ & $-0.0495 \pm 0.0132$ & -0.236 & 0.813 \\
\hline & $\mathrm{L}$ & $-0.0500 \pm 0.1821$ & $-0.0487 \pm 0.0142$ & -0.026 & 0.979 \\
\hline
\end{tabular}

Table 3. $\mathrm{R} 2^{*}$ value $(\overline{\mathrm{X}} \pm \mathrm{s})$ of each $\mathrm{ROI}$ in $\mathrm{AD}$ and control group and Mann-Whitney U test.

\begin{tabular}{|c|c|c|c|c|c|}
\hline & ROI & $\mathrm{AD}$ & Control & $Z$ value & $\mathrm{P}$ value \\
\hline \multirow[t]{2}{*}{ HP } & $\mathrm{R}$ & $20.332 \pm 4.049$ & $18.675 \pm 4.273$ & -2.039 & 0.041 \\
\hline & $\mathrm{L}$ & $21.782 \pm 3.729$ & $17.550 \pm 3.086$ & -3.133 & 0.001 \\
\hline \multirow[t]{2}{*}{ GP } & $\mathrm{R}$ & $57.786 \pm 16.305$ & $46.327 \pm 12.472$ & -2.237 & 0.025 \\
\hline & $\mathrm{L}$ & $56.608 \pm 18.450$ & $49.605 \pm 18.089$ & -1.888 & 0.059 \\
\hline \multirow[t]{2}{*}{ PUT } & $\mathrm{R}$ & $38.688 \pm 8.631$ & $33.290 \pm 8.248$ & -2.216 & 0.027 \\
\hline & $\mathrm{L}$ & $38.744 \pm 10.149$ & $31.949 \pm 7.961$ & -2.148 & 0.032 \\
\hline \multirow[t]{2}{*}{ CAU } & $\mathrm{R}$ & $20.002 \pm 5.142$ & $17.604 \pm 3.068$ & -2.043 & 0.039 \\
\hline & $\mathrm{L}$ & $20.873 \pm 3.687$ & $17.112 \pm 4.237$ & -3.142 & 0.001 \\
\hline \multirow[t]{2}{*}{$\mathrm{DN}$} & $\mathrm{R}$ & $41.133 \pm 5.840$ & $38.567 \pm 7.134$ & -0.752 & 0.452 \\
\hline & $\mathrm{L}$ & $42.440 \pm 6.476$ & $37.739 \pm 7.833$ & -1.587 & 0.112 \\
\hline \multirow[t]{2}{*}{$\mathrm{SN}$} & $\mathrm{R}$ & $42.846 \pm 10.363$ & $40.159 \pm 4.823$ & -0.315 & 0.753 \\
\hline & $\mathrm{L}$ & $43.284 \pm 11.210$ & $40.053 \pm 6.079$ & 0.410 & 0.681 \\
\hline \multirow[t]{2}{*}{$\mathrm{RN}$} & $\mathrm{R}$ & $39.300 \pm 8.696$ & $37.305 \pm 5.077$ & -0.506 & 0.613 \\
\hline & $\mathrm{L}$ & $37.917 \pm 9.454$ & $37.484 \pm 4.221$ & -1.095 & 0.274 \\
\hline \multirow[t]{2}{*}{$\mathrm{TH}$} & $\mathrm{R}$ & $19.993 \pm 4.121$ & $19.948 \pm 4.045$ & -0.192 & 0.819 \\
\hline & $\mathrm{L}$ & $22.098 \pm 2.853$ & $21.579 \pm 1.878$ & -0.315 & 0.799 \\
\hline
\end{tabular}


from 0.531 to 0.433 (Table 4). The phase values of the left PUT had the most significant correlation with the

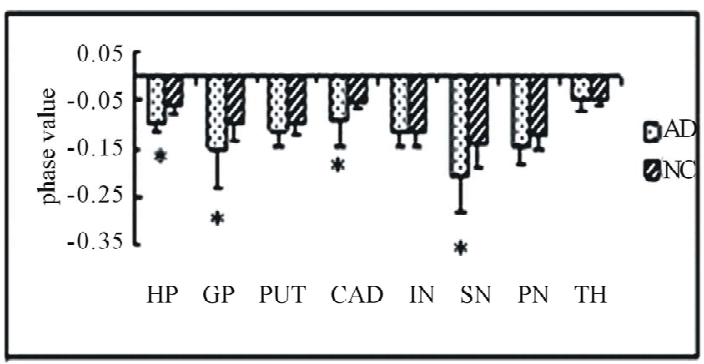

(a)

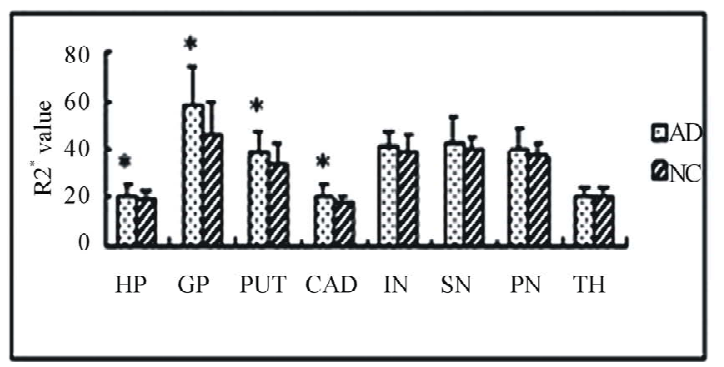

(c)
MMSE scores in AD group, the correlation coefficient was $0.531(\mathrm{p}=0.012)$. The correlation coefficient be-

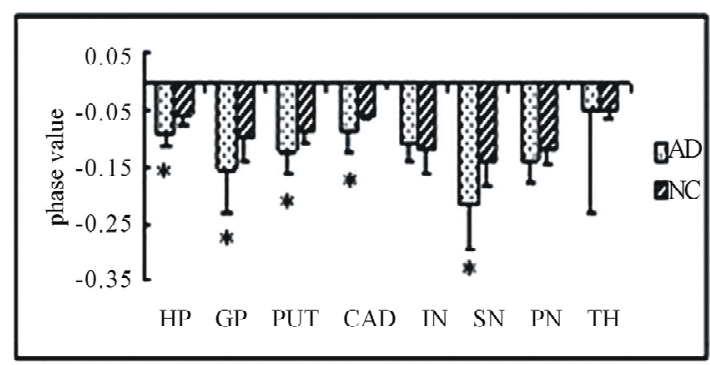

(b)

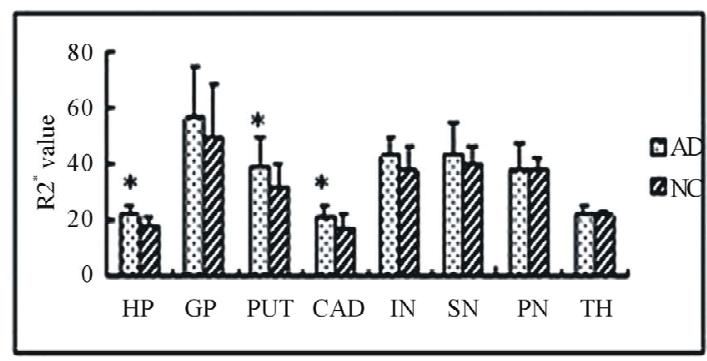

(d)

Figure 3. Caption: Plot of phase value of each brain subregion between $\mathrm{AD}$ and control groups for right (a) and left (b) hemisphere. Plot of $\mathrm{R}^{*}$ value of each brain subregion between $\mathrm{AD}$ and $\mathrm{NC}$ groups for right (c) and left (d) hemisphere. Note: ${ }^{*}=$ significant difference of phase value and $\mathrm{R} 2^{*}$ value between $\mathrm{AD}$ and NC group in each ROI.

Table 4. Correlation test between phase values and MMSE scores, $\mathrm{R} 2^{*}$ values and MMSE scores in AD group.

\begin{tabular}{|c|c|c|c|c|c|}
\hline & ROI & Phase value & & $\mathrm{R} 2^{*}$ value & \\
\hline & & $\mathrm{r}$ & P value & $\mathrm{r}$ & $P$ value \\
\hline \multirow[t]{2}{*}{$\mathrm{HP}$} & $\mathrm{R}$ & 0.321 & 0.145 & 0.246 & 0.271 \\
\hline & $\mathrm{L}$ & 0.435 & 0.043 & -0.424 & 0.049 \\
\hline \multirow[t]{2}{*}{ GP } & $\mathrm{R}$ & 0.476 & 0.025 & -0.414 & 0.055 \\
\hline & $\mathrm{L}$ & 0.509 & 0.016 & -0.380 & 0.081 \\
\hline \multirow[t]{2}{*}{ PUT } & $\mathrm{R}$ & 0.433 & 0.044 & -0.354 & 0.106 \\
\hline & $\mathrm{L}$ & 0.531 & 0.012 & -0.521 & 0.013 \\
\hline \multirow[t]{2}{*}{ CAU } & $\mathrm{R}$ & 0.434 & 0.044 & -0.253 & 0.256 \\
\hline & $\mathrm{L}$ & 0.32 & 0.146 & -0.369 & 0.091 \\
\hline \multirow[t]{2}{*}{$\mathrm{DN}$} & $\mathrm{R}$ & 0.444 & 0.039 & -0.042 & 0.851 \\
\hline & $\mathrm{L}$ & 0.212 & 0.322 & -0.061 & 0.786 \\
\hline \multirow[t]{2}{*}{$\mathrm{SN}$} & $\mathrm{R}$ & 0.095 & 0.675 & 0.072 & 0.751 \\
\hline & $\mathrm{L}$ & -0.003 & 0.99 & 0.112 & 0.620 \\
\hline \multirow[t]{2}{*}{$\mathrm{RN}$} & $\mathrm{R}$ & 0.209 & 0.35 & -0.188 & 0.402 \\
\hline & $\mathrm{L}$ & 0.268 & 0.227 & -0.031 & 0.892 \\
\hline \multirow[t]{2}{*}{$\mathrm{TH}$} & $\mathrm{R}$ & 0.323 & 0.143 & -0.378 & 0.083 \\
\hline & $\mathrm{L}$ & 0.262 & 0.238 & 0.023 & 0.919 \\
\hline
\end{tabular}


tween the phase values of the left HP and the MMSE scores in AD group were $0.435(p=0.043)$. The $R 2^{*}$ values of the left PUT and left HP were negatively correlated with MMSE scores, the correlation coefficient was $-0.521(\mathrm{p}=0.013)$ and $-0.424(\mathrm{p}=0.049)$ respectively

(Table 4, Figure 4).

\section{DISCUSSION}

An imbalance in iron homeostasis is a precursor of the neurodegenerative processes leading to $\mathrm{AD}$ and iron deposition disturbance in $\mathrm{AD}$ brain have been proved in histological analysis and in animal models [23]. Animal models showed increased iron deposition in HP, parietal cortex and motor cortex in AD brain [24-26]. Higher iron concentrations in the AD brain may increase the oxidation in HP, frontal cortex, entorhinal cortex, PUT, and amygdala [27-28]. Iron imbalance is not necessarily restricted to the affected regions but can also affect the cortex and cerebellum in pre-clinical AD and mild cognitive impairment (MCI) cases [29].

In our research, the change in the phase values and R2 ${ }^{*}$ values were compatible with the most vulnerable

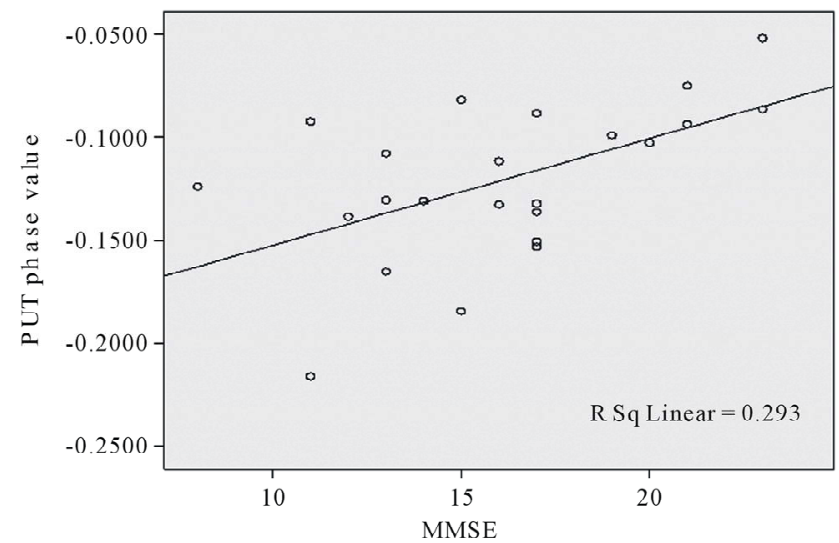

(a)

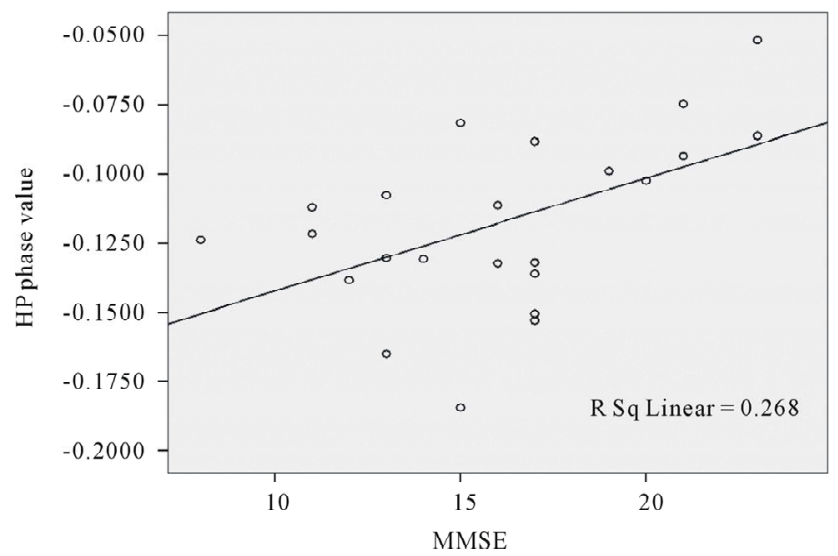

(c) regions of the $\mathrm{AD}$ brain. Several $\mathrm{MR}$ techniques revealed increased iron deposition in some sub regions of $\mathrm{AD}$ brain; HP, cortex, CAU, GP, and PUT [30-32]. Phase imaging research [33] established the significance increase of iron deposition in bilateral body of HP, entorhinal cortex, frontal cortex, head of CAU, and PUT in AD groups. Another study [21] demonstrated statistically lower phase values in bilateral HP, CAU, DN, PUT, parietal cortex in $\mathrm{AD}$ group. In $\mathrm{AD}$ brain, $\mathrm{R} 2$ in the grey matter of temporal lobe increased notably [3]. However, the results among these studies were not exactly the same. This could be due to the differences in inclusion criteria, imaging protocol, field strength, post-processing methods, and the definition of the ROIs. The whole process of iron deposition in AD brain and its relationship with the progression of AD is still unclear. Data from quantitative histopathological prospective studies are required to establish the relationship.

The paramagnetic nature of iron can increase $\mathrm{R} 2^{*}$ effects which can be semi-quantitatively assessed by measuring $\mathrm{R} 2^{*}$ values and the phase shift in vivo. Limited research has been conducted on comparison of two

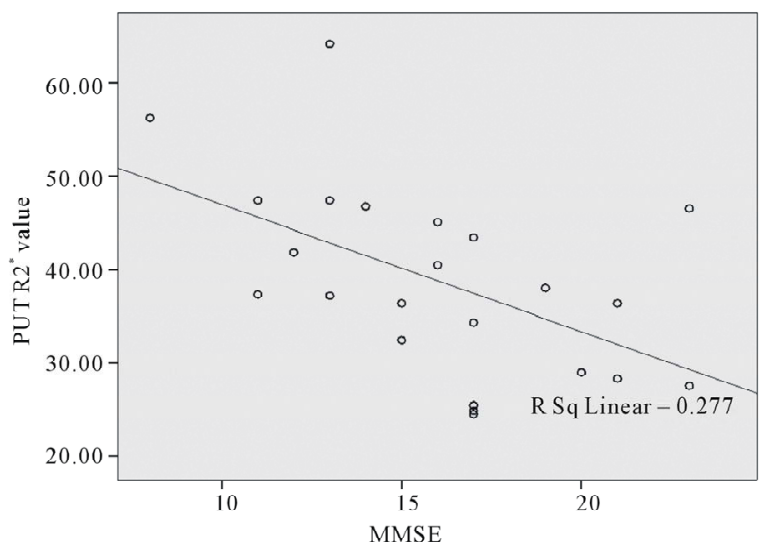

(b)

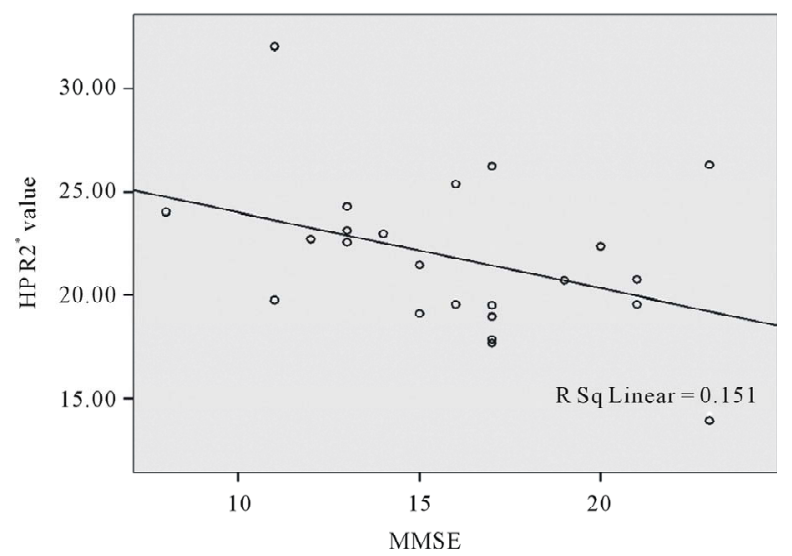

(d)

Figure 4. Caption: Correlation of MMSE scores to phase value (a) and R2 ${ }^{*}$ value (b) in left PUT in AD group, Correlation of MMSE scores phase value (c) and $\mathrm{R} 2^{*}$ value (d) in left HP in AD group. 
methods $[18,19,35]$. In our study, the iron content in various brain regions in two groups showed inconstant phase values compared to the results obtained from the $\mathrm{R} 2^{*}$ values. However, $\mathrm{R} 2^{*}$ values of bilateral (L\&R) SN did not show any difference in two groups. A recent study showed that the phase is more sensitive to iron even in smaller amounts, whereas $\mathrm{T} 2{ }^{*}$ undervalue the iron content in smaller amounts [35]. The SN is a relatively smaller brain structure compared to other ROIs, therefore only phase showed increased iron in AD group. Though the phase value of right PUT and the $\mathrm{R} 2{ }^{*}$ values of the left GP did not achieve statistical significance level, the iron content of bilateral PUT and GP in AD group showed increased based on phase and $\mathrm{R} 2^{*}$ values.

Both the methods have important features. Phase values are influenced by the deoxyhaemoglobin than $\mathrm{R} 2^{*}$ value, both blood volume and higher ferritin content contribute in the contrast of grey and white matter on phase imaging [36]. Spatial filtering could alter the true phase shifts based on the size and shape of the anatomical structure; it would reduce the apparent phase shift in large uniform structures, such as CAU, but had little effect on smaller structures, such as SN [15]. In addition to the iron or other paramagnetic substances, $\mathrm{R} 2{ }^{*}$ values can be affected by water content, gliosis, closely arranged myelinated nerve fibers, etc. [10,34,36]. Both iron and myelin can increase $\mathrm{R} 2^{*}$. Alzheimer patients are accompanied by cortical atrophy, gliosis, and demyelinating disorders. Moreover there is an increase in water concentration in AD brain, especially in the HP region, which is more vulnerable to the expansion of the temporal horn of lateral ventricle. This may result in a reducetion in $\mathrm{R} 2{ }^{*}$ values, and consequently the increasing effect of $\mathrm{R} 2^{*}$ value by iron deposition would be weakened. To reduce the impact of these factors we have applied higher field strength (3T) to provide high sensitivity of $\mathrm{R} 2^{*}$ value to iron [34]. All these impact factors mentioned might account for the differences between the two methods. Earlier study showed high contrast SWI images in plaques of transgenic mouse brain than $\mathrm{R} 2{ }^{*}$ images [19], however there is no quantitative comparison of phase value and $\mathrm{R} 2^{*}$ value in $\mathrm{AD}$ brain.

Phase values of ROIs showed more correlation with MMSE than $\mathrm{R} 2{ }^{*}$ values and correlation coefficient was higher in the same ROI (Table 4). The phase value of bilateral GP, PUT, left HP, right CAU and right DN in AD patients were correlated with MMSE scores, respectively (Table 4). According to the previous study [24-28], these ROIs showed disturbance of iron deposition in $\mathrm{AD}$ brain and leads to a conclusion that phase value were more sensitive to the change of $\mathrm{AD}$ related iron deposition. This provides potential applications to the longitudinal monitoring and therapeutic developments for $\mathrm{AD}$.
The phase values and $\mathrm{R} 2^{*}$ values of the left PUT had the most significant correlation with the MMSE scores in AD patients. Multiple studies using SWI phase values showed significantly increased rate of iron uptake in the left PUT in individuals who progressed from MCI to dementia [37]. Increasing left PUT iron levels have been described previously in clinically depressed elderly patients [38]. Depression commonly precedes and may overlap with Alzheimer's type of dementia development [39]. Depression is not only a clinical symptom, but also an important risk factor of $\mathrm{AD}$. Late-onset $\mathrm{AD}$ is always combined with a history of anti-depression treatment. However, the relationship between these two diseases is still ambiguous.

In $\mathrm{AD}$ brain, neurodegeneration in medial temporal lobe is more at early stages [40]. The left hemisphere is dominant and more sensitive to AD than the right side, and the atrophy of left HP is more severe than the right [41]. It has been proved that redox active ions induce neuronal apoptosis and results in brain atrophy. This provides a rational enlightenment to our findings that the phase values and the $\mathrm{R} 2^{*}$ values of the left HP are closely related with the MMSE scores. From another study [33] the phase value of the head of right HP had a moderate positive correlation with MMSE scores. In our study, we did not divide HP into sub regions because of the severe atrophy of $\mathrm{AD}$ brain. These variations in the selection of ROI may explain the different outcomes of the two studies.

The current study has following limitations: MCI patients and patients with the poor image quality were excluded from the study. Few patients were uncertain about the duration so we could not calculate the correlation of iron deposition and the duration of AD. Also we could not figure out the iron deposition in $\mathrm{AD}$ brain between the early-onset (onset age $<65 \mathrm{y} / \mathrm{o}$ ) and late-onset of $\mathrm{AD}$ (onset age $>65 \mathrm{y} / \mathrm{o}$ ). In addition, statistical significance of phase values and $\mathrm{R} 2{ }^{*}$ values in brain sub regions were not entirely consistent between $\mathrm{AD}$ and control groups. Our further study will focus on these problems.

In conclusion, our study results demonstrate the exploitation of SWI phase value and $\mathrm{R} 2{ }^{*}$ values for effecttive examination of abnormal iron deposition in $\mathrm{AD}$ brain, wherein phase has advantages in examination of small brain structure. Phase value showed a high correlation coefficient with MMSE scores and the iron deposition of left PUT has a close relationship with the progression of $\mathrm{AD}$.

\section{ACKNOWLEDGEMENTS}

The study was funded by the Scientific Research Program on Health Care, Ministry of Health (No. 200802099), and Shanghai Science and Technology Commission (No. 11ZR1404500). We extend our sincere thanks to BioQuest for their valuable editorial guidance in the genera- 
tion of this report.

\section{REFERENCES}

[1] Stankiewicz, J, Panter, S.S., Neema, M., et al. (2007) Iron in chronic brain disorders: Imaging and neurotherapeutic implications. Neurotherapeutics, 4, 371-386.

doi:10.1016/j.nurt.2007.05.006

[2] Perry, G., Nunomura, A., Hirai, K., et al. (2000) Oxidative damage in Alzheimer's disease: The metabolic dimension. International Journal of Developmental Neuroscience, 18, 417-421. doi:10.1016/S0736-5748(00)00006-X

[3] Nunomura, A., Perry, G., Aliev, G., et al. (2001) Oxidative damage is the earliest event in Alzheimer disease. Journal of Neuropathology \& Experimental Neurology, 60, 759767.

[4] Atwood, C.S., Obrenovich, M.E., Liu, T.B., et al. (2003) Amyloid- $\beta$ : A chameleon walking in two worlds: A review of the trophic and toxic properties of amyloid- $\beta$. Brain Research Reviews, 43, 1-16. doi:10.1016/S0165-0173(03)00174-7

[5] Schubert, D. and Chevion, M. (1995) The role of iron in beta amyloid toxicity. Biochemical and Biophysical Research Communications, 216, 702-707. doi:10.1006/bbrc. 1995.2678

[6] Siemonsen, S., Finsterbusch, J., Matschke, J., et al. (2008) Age-dependent normal values of $\mathrm{T} 2 *$ and $\mathrm{T} 2$ ' in brain parenchyma. American Journal of Neuroradiology, 29, 950-955. doi:10.3174/ajnr.A0951

[7] Peran, P., Hagberg, G., Luccichenti, G., et al. (2007) Voxel-based analysis of R2* maps in the healthy human brain. Journal of Magnetic Resonance Imaging, 26, 14131420. doi:10.1002/jmri.21204

[8] Jensen, J.H., Szulc, K., Hu, C.X., et al. (2009) Magnetic field correlation as a measure of iron-generated magnetic field inhomogeneities in the brain. Magnetic Resonance in Medicine, 61, 481-485. doi:10.1002/mrm.21823

[9] Bartzokis, G., Tishler, T.A., Lu, P.H., et al. (2007) Brain ferritin iron influence age- and gender-related risks of neurodegeneration. Neurobiology of Aging, 28, 414-423. doi:10.1016/j.neurobiolaging.2006.02.005

[10] Yao, B., Li, T.Q., Van Gelderen, P., et al. (2009) Susceptibility contrast in high field MRI of human brain as a function of tissue iron content. Neuroimage, 44, 12591266. doi:10.1016/j.neuroimage.2008.10.029

[11] Xu, X.J., Wang, Q.D. and Zhang, M.M. (2008) Age, gender, and hemispheric differences in iron deposition in the human brain: An in vivo MRI study. Neuroimage, 40, 35-42. doi:10.1016/j.neuroimage.2007.11.017

[12] Harder, S.L., Hopp, K.M., Ward, H., et al. (2008) Mineralization of the deep gray matter with age: A retrospective review with susceptibility-weighted MR imaging. American Journal of Neuroradiology, 29, 176-183. doi:10.3174/ajnr.A0770

[13] Haacke, E.M., Cheng, N.Y., House, M.J., et al. (2005) Imaging iron stores in the brain using magnetic resonance imaging. Journal of Magnetic Resonance Imaging, 23, 1-25. doi:10.1016/j.mri.2004.10.001

[14] Pfefferbaum, A., Adalsteinsson, E., Rohlfing, T., et al. (2009) MRI estimates of brain iron concentration in normal aging: Comparison of field-dependent (FDRI) and phase(SWI) methods. NeuroImage, 47, 493-500. doi:10.1016/j.neuroimage.2009.05.006

[15] Ogg, R.J., Langston, J.W., Haacke, E.M., et al. (1999) The correlation between phase shifts in gradient-echo MR images and regional brain iron concentration. Journal of Magnetic Resonance Imaging, 17, 1141-1148. doi:10.1016/S0730-725X(99)00017-X

[16] Haacke, E.M., Xu, Y.B., Cheng, Y.C.N., et al. (2004) Susceptibility weighted imaging (SWI). Magnetic Resonance in Medicine, 52, 612-618. doi:10.1002/mrm.20198

[17] McCrea, R.P., Harder, S.L., Martin, M., et al. (2008) A comparison of rapid-scanning X-ray fluorescence mapping and magnetic resonance imaging to localize brain iron distribution. European Journal of Radiology, 68, S109-S113. doi:10.1016/j.ejrad.2008.04.048

[18] Haacke, E.M., Ayaz, M., Khan, A., et al. (2007) Establishing a baseline phase behavior in magnetic resonance imaging to determine normal vs. abnormal iron content in the brain. Journal of Magnetic Resonance Imaging, 26, 256-264. doi:10.1002/jmri.22987

[19] Chamberlain, R., Reyes, D., Curran, G.L., et al. (2009) Comparison of amyloid plaque contrast generated by T-2-weighted, T-2(star)-weighted, and susceptibility-weighted imaging methods in transgenic mouse models of Alzheimer's disease. Magnetic Resonance in Medicine, 61, 1158-1164. doi:10.1002/mrm.21951

[20] Meadowcroft, M.D., Connor, J.R., Smith, M.B., et al. (2009) MRI and histological analysis of beta-amyloid plaques in both human Alzheimer's disease and APP/PS1 transgenic mice. Journal of Magnetic Resonance Imaging, 29, 997-1007. doi:10.1002/jmri.21731

[21] Zhu, W.Z., Zhong, W.D., Wang, W., et al. (2009) Quantitative MR phase-corrected imaging to investigate increased brain iron deposition of patients with Alzheimer disease. Radiology, 253, 497-504. doi:10.1148/radiol.2532082324

[22] McKhann, G., Drachman, D., Folstein, M., et al. (1984) Clinical diagnosis of Alzheimer's disease: Report of the NINCDS-ADRDA Work Group under the auspices of Department of Health and Human Services Task Force on Alzheimer's disease. Neurology, 34, 939-944. doi:10.1212/WNL.34.7.939

[23] Berg, D. and Youdim, M.B. (2006) Role of iron in neurodegenerative disorders. Topics in Magnetic Resonance Imaging, 17, 5-17. doi:10.1097/01.rmr.0000245461.90406.ad

[24] Good, P.F., Perl, D.P., Bierer, L.M., et al. (1992) Selective accumulation of aluminum and iron in the neurofibrillary tangles of Alzheimer's disease: A laser microprobe (LAMMA) study. Annals of Neurology, 31, 286-292. doi:10.1002/ana.410310310

[25] Dedman, D.J., Treffry, A., Candy, J.M., et al. (1992) Iron and aluminium in relation to brain ferritin in normal indi- 
viduals and Alzheimer's disease and chronic renal-dialysis patients. Biochemical Journal, 287, 509-514.

[26] Connor, J.R., Snyder, B.S., Beard, J.L., et al. (1992) Regional distribution of iron and iron-regulatory proteins in the brain in aging and Alzheimer's disease. Journal of Neuroscience Research, 31, 327-335. doi:10.1002/jnr.490310214

[27] Loeffler, D.A., Lewitt, P.A., Juneau, P.L., et al. (1996) Increased regional brain concentrations of ceruloplasmin in neurodegenerative disorders. Brain Research, 738, 265-274. doi:10.1016/S0006-8993(96)00782-2

[28] Pappolla, M.A., Omar, R.A., Kim, K.S., et al. (1992) Immunohistochemical evidence of antioxidant stress in Alzheimer's disease. American Journal of Pathology, 140, 621-628.

[29] Smith, M.A., Zhu, X.W., Tabaton, M., et al. (2010) Increased iron and free radical generation in preclinical Alzheimer disease and mild cognitive impairment. Journal of Alzheimer's Disease, 19, 363-372.

[30] Bartzokis, G., Tishler, T.A., Shin, I.S., et al. (2004) Brain ferritin iron as a risk factor for age at onset in neurodegenerative diseases. Annals of the New York Academy of Sciences, 1012, 224-236. doi:10.1196/annals.1306.019

[31] Press, D.Z., Casement, M.D., Schenck, J.F., et al. (2004) High field MRI demonstrates increased brain iron in Alzheimer's disease. Neurobiology of Aging, 25, S378-S379. doi:10.1016/S0197-4580(04)81240-5

[32] Bartzokis, G., Sultzer, D., Cummings, J., et al. (2000) In vivo evaluation of brain iron in Alzheimer Disease using magnetic resonance imaging. Archives of General Psychiatry, 57, 47-53. doi:10.1001/archpsyc.57.1.47

[33] Ding, B., Chen, K.M., Ling, H.W., et al. (2009) Correlation of iron in the hippocampus with MMSE in patients with Alzheimer's disease. Journal of Magnetic Resonance Imaging, 29, 793-798. doi:10.1002/jmri.21730

[34] House, M.J., Pierre, T.G.S., and McLean, C. (2008) 1.4T study of proton magnetic relaxation rates, iron concentrations, and plaque burden in Alzheimer's disease and control postmortem brain tissue. Magnetic Resonance in Medicine, 60, 41-52. doi:10.1002/mrm.21586

[35] Haacke, E.M., Miao, Y.W., Liu, M.J., et al. (2010) Correlation of putative iron content as represented by changes in R2* and phase with age in deep gray matter of healthy adults. Journal of Magnetic Resonance Imaging, 32, 561576. doi:10.1002/jmri.22293

[36] Duyn, J.H., Van Gelderen, P., Li, T.Q., et al. (2007) High-field MRI of brain cortical substructure based on signal phase. Proceedings of the National Academy of Sciences of the United State of America, 104, 1179611801. doi:10.1073/pnas.0610821104

[37] Kirsch, W., McAuley, G., Holshouser, B., et al. (2009) Serial susceptibility weighted MRI measures brain iron and microbleeds in dementia. Journal of Alzheimer's Disease, 17, 599-609.

[38] Steffens, D.C., Tupler, L.A., Ranga, K., et al. (1998) Magnetic resonance imaging signal hypointensity and iron content of putamen nuclei in elderly depressed patients. Journal of Psychiatric Research, 83, 95-103. doi:10.1016/S0925-4927(98)00032-8

[39] Visser, P.J., Verhey, F.R.J., Ponds, R.W.H.M., et al. (2000) Distinction between preclinical Alzheimer's disease and depression. Journal of the American Geriatrics Society, 48, 479-484.

[40] Sluimer, J.D., Van der Flier, W.M., Karas, G.B., et al. (2009) Accelerating regional atrophy rates in the progression from normal aging to Alzheimer's disease. European Radiology, 19, 2826-2833. doi:10.1007/s00330-009-1512-5

[41] Scahill, R.I., Schott, J.M., Stevens, J.M., et al. (2002) Mapping the evolution of regional atrophy in Alzheimer's disease: Unbiased analysis of fluid-registered serial MRI. Proceedings of the National Academy of Sciences of the United State of America, 99, 4703-4707. doi:10.1073/pnas.052587399 